\title{
FIELD TEST AND ANALYSIS: THE BEHAVIOR OF 3-J CONCENTRATOR CELLS UNDER THE CONTROL OF CELL TEMPERATURE
}

\author{
H.S. Lee ${ }^{1 *}$, N.J. Ekins-Daukes ${ }^{1}$, K. Araki $^{2}$, Y. Kemmoku ${ }^{3}$, M. Yamaguchi ${ }^{1}$ \\ 1. Toyota Technological Institute, 2-12-1 Hisakata, Tempaku-ku, Nagoya 468-8511, Japan \\ 2. Daido Steel Co., Ltd. Nagoya, Japan \\ 3. Toyohashi Sozo College, Toyohashi, Japan
}

\begin{abstract}
The behavior of 3-junction concentrator cells (InGaP/(In)GaAs/Ge) under the control of cell temperature is investigated and evaluated at Toyota Technological Institute of Japan. The aim of this study is to investigate an accurate temperature coefficient as comparing the outdoor test with the theoretical calculation as a function of a sunlight concentration. Recently, the field test consisted of a total of 6 modules at two sites (Inuyama, Toyohashi) of Japan shows the peak efficiency of $26.8 \% \pm 1.5 \%$, and the $3-\mathrm{J}$ cells show the efficiency of $38.9 \%$ at 489 suns. In this study, a new dome-typed Fresnel lens (550X) that has the optical efficiency of $85.8 \%$ was used. The homogenizer is placed on the holder designed to control the cell temperature, and the outdoor test using the solar tracker was carried out under the 400X, 550X lenses. The conversion efficiency increases with the direct solar radiation, and decreases as increasing the receiver temperature. However, no change in Isc was observed, and $V_{O C}$ decreased slightly as a function of the receiver temperatures. The temperature coefficient obtained from the receiver at TTI shows the similar trends with them from the bare cell at Nara-AIST.
\end{abstract}

\section{III-V MJ CONCENTRATOR CELLS AND MODULES}

The concentrator modules using the III-V multijunction (MJ) solar cells $(\operatorname{InGaP} /(\ln ) \mathrm{GaAs} / \mathrm{Ge})$ have been developed [1], and expected to reduce the PV cost. The

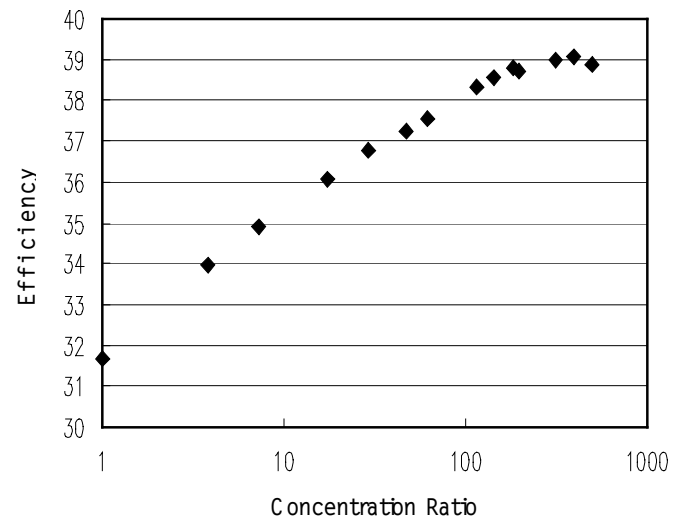

Fig. 1. The relationships between the cell efficiency and number of suns presented in 19-EUPVSEC.

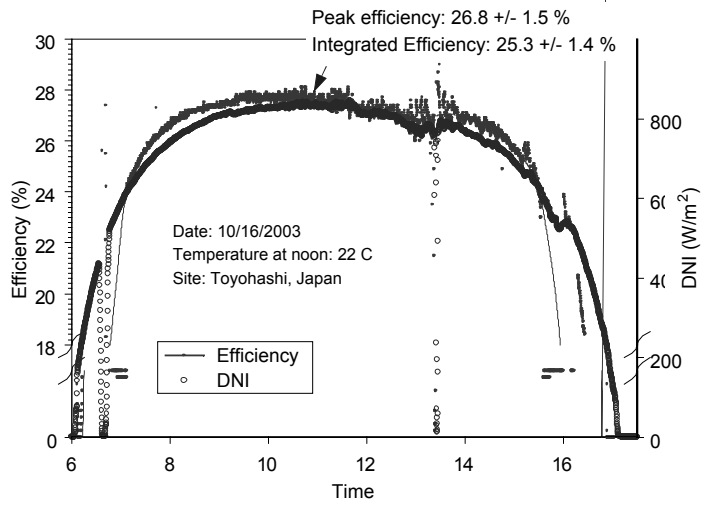

Fig. 2. Power generation measured at Toyohashi for a sunny day.

efficiency of $38.9 \%$ at 489 suns has been attained successfully in the concentrator $\mathrm{InGaP} /(\mathrm{In}) \mathrm{GaAs} / \mathrm{Ge} 3-\mathrm{J}$ cells with the double hetero structure of tunnel junction, as seen in Fig. 1. In addition, a new dome-typed Fresnel lens has been developed, and recently, the optical efficiency of $85.8 \%$ was obtained at $550 \mathrm{X}$ lens which was made by the injection molding method. From these developments of the concentrator cells and lenses, they are expected to replace the silicon concentrator cells that have been widely used for the large-scale solar concentrators.

We have reported the field test that is carried out using 400X module $\left(7,056 \mathrm{~cm}^{2}\right)$ with 36 solar cells in two sites of Japan (Inuyama, Toyohashi) for a long term. The averaged efficiency has been obtained as $27.9 \%$, corresponding $28.1 \%$ after the module temperature correction to $25^{\circ} \mathrm{C}$, as seen in Fig. 2. The peak efficiency is $26.8 \% \pm 1.5 \%$ after considering the measurements errors of the direct normal irradiance. Recently, the new concentrator 550X modules $\left(5,445 \mathrm{~cm}^{2}\right)$ consisted of 20 solar cells were developed, and their efficiencies were $28.9 \%$, corresponding $31.5 \pm 2 \%$ after the temperature correction in the best sunshine conditions.

\section{THE OUTDOOR TEST AT TTI}

A new field test has started at Toyota Technological Institute (TTI), Nagoya to measure the temperature coefficients in a different concentration ratio. In this study, the behavior of the receiver consisted 3-J cell with 400X and 550X lenses under the control of the temperature is 
investigated. The accurate analysis of the temperature coefficient in the 3-J concentrator cells under the concentrated sunlight is aim of this study to improve the performance of the concentrator cells and modules.

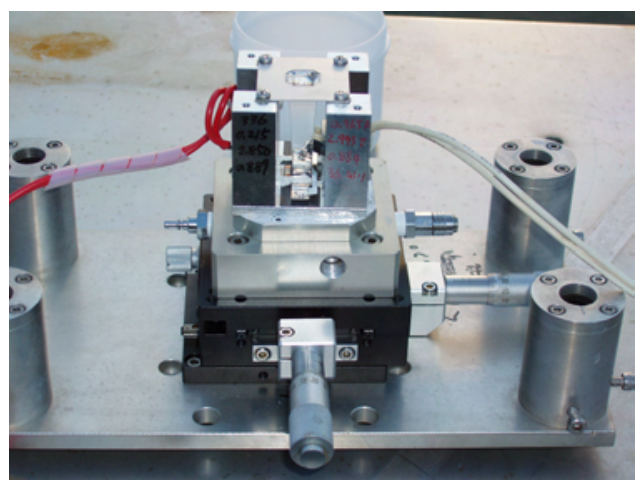

Fig. 3. The assembly of homogenizer placed on the holder.

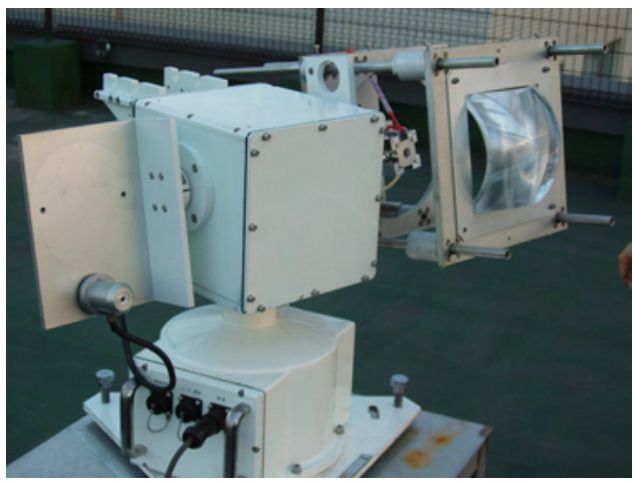

Fig. 4. The outdoor test using the solar tracker.

A new dome-typed Fresnel lens (550X) that has the optical efficiency of $85.8 \%$ has been developed [2], and is used in this study. The homogenizer is placed on the holder designed to control the cell temperature, as seen in Fig. 3. The outdoor test is performed by using the solar tracker under the concentration of sunlight [Fig. 4]. The temperatures of the receiver are measured by using thermocouples.

\section{THE TEMPERATURE COEFFICIENT}

The relationships between the direct solar radiation (a) and conversion efficiency $(\eta)$, and $\eta(b), I_{S C}(c), V_{O C}(d)$ and the temperatures of receiver with $3-\mathrm{J}$ concentrator cell measured in TTI under the geometrical concentration ratio of 400X are shown in Fig. 5. To investigate the effects of the receiver temperature, $\eta, I_{S C}$, and $V_{O C}$ were measured at a specific solar radiation $\left(0.5 \mathrm{~kW} / \mathrm{m}^{2}\right)$. As seen in Fig. 5 , the conversion efficiency increases with the direct solar radiation. In addition, the conversion efficiency decreases as increasing the receiver temperature, where the loss of efficiency is around $0.5 \%$ as the receiver temperature rises by $1^{\circ} \mathrm{C}$. However, no change in
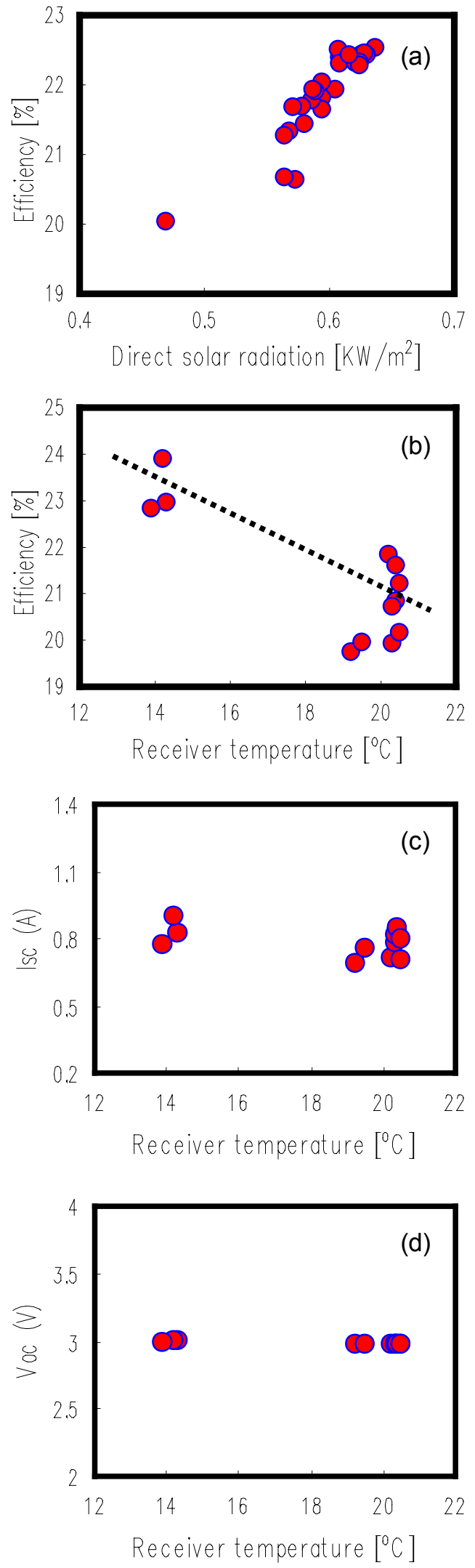
Fig. 5. Relationships between the direct solar radiation (a) and efficiency $(\eta)$, and $\eta(b), I_{S C}(c), V_{O C}(d)$ vs the temperatures of receiver with $3-\mathrm{J}$ concentrator cell (400X).
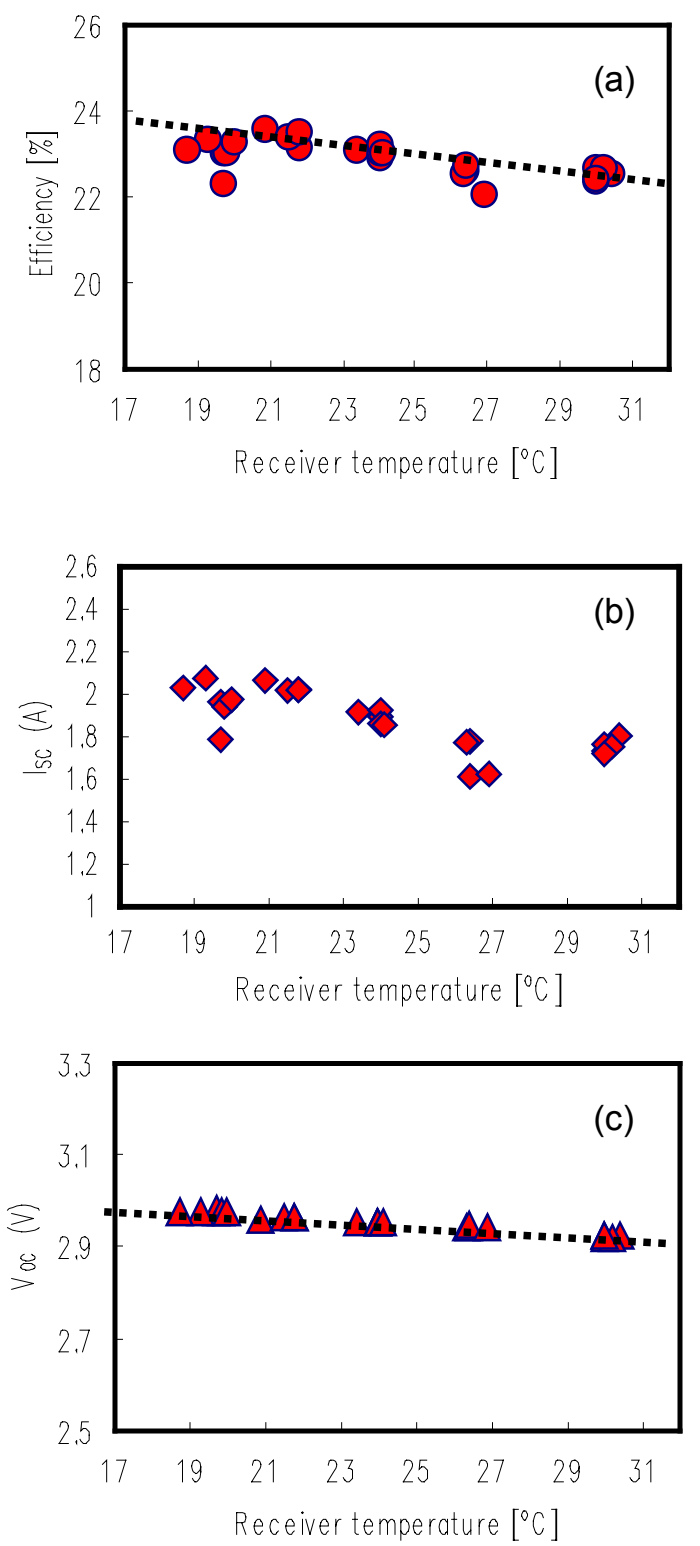

Fig. 6. Relationships between $\eta(a), I_{S C}(b), V_{O C}(c)$ and the receiver temperatures under the concentration ratio of $550 \mathrm{X}$.

$I_{S C}$ (c) has been observed, and $V_{O C}$ decreased slightly as a function of the receiver temperatures. Figure 6 shows the relationships between efficiency $(\eta)(a), I_{S C}(b), V_{O C}(c)$ and the receiver temperatures under the concentration ratio of $550 \mathrm{X}$ measured at a direct solar radiation $\left(\sim 0.8 \mathrm{~kW} / \mathrm{m}^{2}\right)$. As seen in Fig. 6, the conversion efficiency and $V_{O C}$ decrease slightly with the receiver temperatures. In this study, to understand the temperature coefficient between the module and cell, we have also observed the cell temperature because the cell temperature in concentrator module is not always in agreement with the module temperature, as seen in Fig. 7. The temperature of cell rises with the product of heat flux and resistance. The temperature of cell was measured with a forward bias heating, and increased linearly.

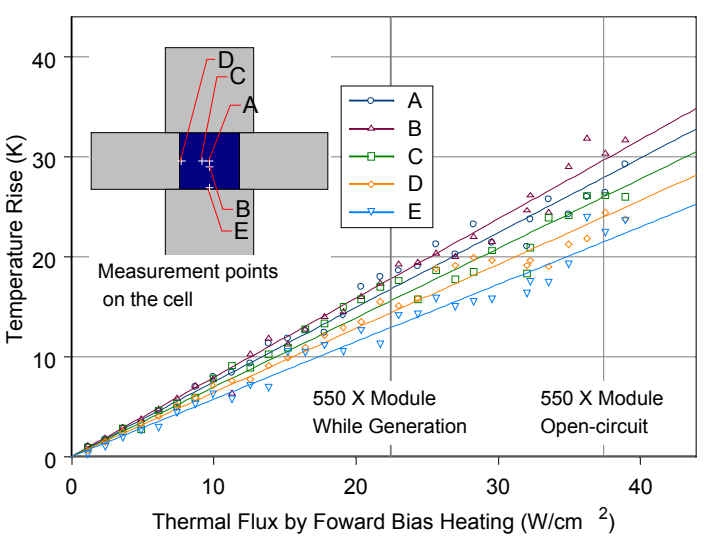

Fig. 7. Changes of the cell temperatures as a function of thermal flux by a forward bias heating.

The temperature coefficient measured at TTI is shown in Fig. 8, in comparison with the results measured by another group. The temperature coefficient decreases with increasing the concentration ratio and short-circuit current. The calculated value was obtained from the temperature coefficient calculated in previous report [3] under the illumination of 1-sun. The values obtained at the NaraAIST [4] were measured from the bare cell on the temperature-controlled stage, and the values obtained at Toyohashi from the statistical analysis during 1 year mission with the concentrator module with 32 cells using 400X flat Fresnel lens [5].

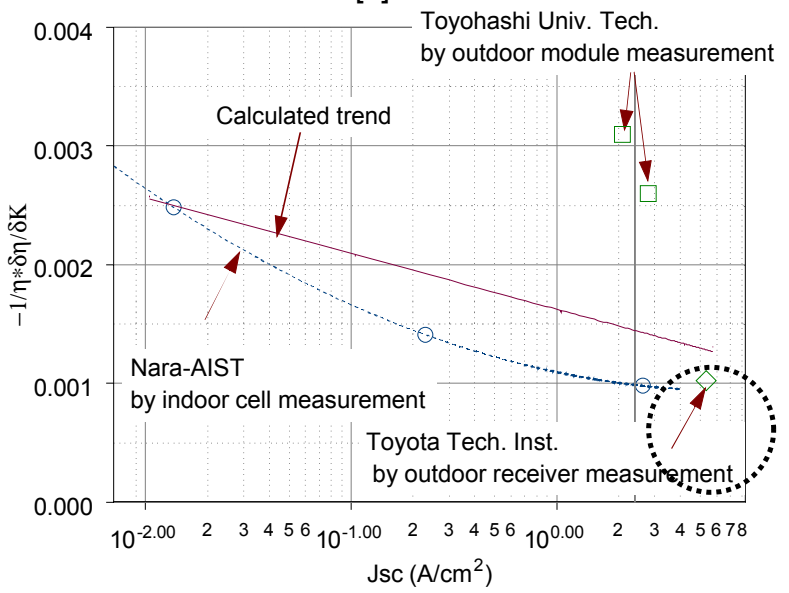

Fig. 8. The temperature coefficient with a short-circuit current measured in Nara-AIST, Toyohashi, TTI of Japan.

As a result, the values obtained from the receiver at TTI shows the similar trends with them from the bare cell at Nara-AIST. However, the temperature coefficient obtained from the module measurements at Toyohashi 
show a different result. More experimental data from the field test is needed to understand the relationships of the temperature coefficient in the bare cell, receiver and module under a various concentration ratio of sunlight.

We will measure and report the changes of temperature coefficient with the different short-circuit current later. In addition, we will report a field test measured by the concentrator modules $(2.5 \mathrm{~m} \times 2.0 \mathrm{~m})$ and the solar flat panel, which will be established later at TTI to test the concentrator system. The production scale of this concentrator system is $1.2 \mathrm{~kW}$, the total weight $300 \mathrm{~kg}$, and the resistances of wind force $60 \mathrm{~m} / \mathrm{s}$.

\section{SUMMARY}

A new concentrator cells (InGaP/(In)GaAs/Ge) with a dome-typed Fresnel lens (550X, 85.8\%) have been developed, and showed the $28.9 \%$ efficiencies, corresponding $31.5 \pm 2 \%$ after the temperature correction in the best sunshine condition (Field test: Inuyama, Toyohashi).

A new field test was carried out at Nagoya (TTI) to determine the correct temperature coefficients of the receiver consisted $3-\mathrm{J}$ cell as a function of the concentration ratio (400X, 550X). The conversion efficiency increases with the direct solar radiation, and decreases as increasing the receiver temperature. However, no change in $I_{S C}$ was observed, and $V_{O C}$ decreased slightly as a function of the receiver temperatures. The temperature coefficient obtained from the receiver at TTI shows the similar trends with them from the bare cell at Nara-AIST. However, the temperature coefficient obtained from the theoretical calculation and outdoor measurements of the modules at Toyohashi show the different results.

More test as a function of concentration ratio is needed to understand the accurate temperature coefficient in the bare cell, receiver and module. We will report the changes of the temperature coefficient as a function of a short-circuit current later.

\section{ACKNOWLEDGMENT}

This work was supported by the Incorporated Administrative Agency New Energy and Industrial Technology Development Organization (NEDO) under Ministry of Economy, Trade and Industry (METI), and partially supported by the Ministry of Education Culture, Sports, Science and Technology as a Private University Academic Frontier Center Program in Japan.

\section{REFERENCES}

[1] K.Araki et al., "Packaging III-V Tandem Solar Cells for Practical Terrestrial Applications Achievable to $28 \%$ of Module Efficiency by Conventional Machine Assemble Technology and possibility of 500X and low weight HCPV for space", 19th EUPVSEC, 2004, pp. 2451-2454.

[2] K. Araki et al., "Development of a new 550X concentrator module with $3 \mathrm{~J}$ cells-Performance and Reliability-", Proc. 31st IEEE PVSC Orlando, 2005.
[3] D. J. Friedman, "Modeling of tandem cell temperature coefficients", Proc. 25th IEEE PVSC, 1996, pp. 89-92.

[4] K. Nishioka, phD Thesis, 2003.

[5] Y. Kemmoku et al., "Modeling of module temperature of a concentrator PV system", 19th EUPVSEC, 2004, pp. 2568-2571. 